\title{
WEAK AND MEASURE-VALUED SOLUTIONS TO EVOLUTIONARY PDES
}

\author{
by J. Málek, J. Nečas, M. Rokyta and M. Ružička
}

\section{A BOOK REVIEW}

\author{
JIONGMIN YONG \\ Fudan University \\ Department of Mathematics \\ Shanghai 200433, People's Republic of China
}

(Received November, 1996; Revised January 1997)

Many basic equations in physics take the following form:

$$
\frac{\partial \mathbf{u}}{\partial t}+\sum_{j=1}^{s} \frac{\partial}{\partial x_{j}} \mathbf{f}_{j}(\mathbf{u})=0
$$

where $x \in \mathbb{R}^{d}, t>0, \mathbf{u}=\left(u_{1}, \ldots, u_{s}\right)$ represents the local density of the investigated quantities and $\mathbf{f}=\left(f_{1}, \ldots, f_{d}\right)$ is the flux vector. Such an equation is often used to describe the conservation of the density $\mathbf{u}$ in the evolution of physical process. Common examples are conservation of mass, balance of momentum, and balance of energy. Thus, (1) is called a conservation law (equation). In the case $s=1,(1)$ becomes a first order hyperbolic PDE, which we may call a scalar conservation law (equation).

After certain preparation, the authors consider the Cauchy problem for the scalar conservation law:

$$
\left\{\begin{array}{cc}
\frac{\partial u}{\partial t}+\nabla \cdot \mathbf{f}(u)=0, & \text { in } \mathbb{R}^{+} \times \mathbb{R}^{d}, \\
u(0, \cdot)=u_{0}, & \text { in } \mathbb{R}^{d},
\end{array}\right.
$$

with $\mathbf{f}=\left(f_{1}, \ldots, f_{d}\right), f_{j} \in C^{1}(\mathbb{R})$ and $u_{0}: \mathbb{R}^{d} \rightarrow \mathbb{R}$. It is well-known that, in general, (2) does not admit a classical solution even if both $\mathbf{f}$ and $u_{0}$ are smooth. Thus, one seeks weak solutions to (2), by which we mean a function $u \in L_{l o c}^{\infty}\left(\mathbb{R}^{+} \times \mathbb{R}^{d}\right)$ satisfying

$$
\int_{0}^{\infty} \int_{\mathbb{R}^{d}}\left(u \varphi_{t}+\langle\mathbf{f}(u), \nabla \varphi\rangle\right) d x d t+\int_{\mathbb{R}^{d}} u_{0}(x) \varphi(0, x) d x=0,
$$

for all $\varphi \in C_{0}^{1}\left(\mathbb{R} \times \mathbb{R}^{d}\right)$. To obtain a weak solution of (2), one natural method is the so-called vanishing of viscosity. It introduces the parabolic perturbation to equation (2), which amounts to the introduction of the viscosity. Thus, one considers the 
following to approximate (2):

$$
\left\{\begin{array}{cc}
\frac{\partial u^{\epsilon}}{\partial t}-\epsilon \Delta u^{\epsilon}+\nabla \cdot \mathbf{f}\left(u^{\epsilon}\right)=0, & \text { in } \mathbb{R}^{+} \times \mathbb{R}^{d} \\
u^{\epsilon}(0, \cdot)=u_{0}, & \text { in } \mathbb{R}^{d}
\end{array}\right.
$$

Problem (4) admits a unique weak solution $u^{\epsilon}$ if $u_{0} \in L^{2}\left(\mathbb{R}^{d}\right) \cap L^{\infty}\left(\mathbb{R}^{d}\right)$ and $\mathbf{f} \in C^{1}(\mathbb{R})^{d}$. Next, one would like to let $\epsilon \rightarrow 0$, hoping to obtain a weak solution $u$ of (2). In doing that, the concept of entropy plays an essential role. A pair of $C^{1}$ functions $(\eta, \mathbf{q})$ is called an entropy-entropy flux pair of the conservation law (2) if $\eta$ is convex and $\eta^{\prime}(u) \mathbf{f}^{\prime}(u)=\mathbf{q}^{\prime}(u)$, for all $u \in \mathbb{R}$. Then a weak solution $u$ of (2) is called an entropy solution if for every entropy-entropy flux pair $(\eta, \mathbf{q})$ of $(2)$, the following entropy inequality holds in the sense of distribution:

$$
\frac{\partial}{\partial t} \eta(u)+\nabla \cdot \mathbf{q}(u) \leq 0
$$

The central result of this part says the following.

Theorem 1: Let $u_{0} \in L^{1}\left(\mathbb{R}^{d}\right) \cap L^{\infty}\left(\mathbb{R}^{d}\right) \cap B V\left(\mathbb{R}^{d}\right)$ and $\mathbf{f} \in C^{1}\left(\mathbb{R}^{d}\right)$. Then (2) admits a unique entropy solution $u \in L^{\infty}\left((0, \infty) \times \mathbb{R}^{d}\right) \cap C\left([0, T] ; L^{1}\left(\mathbb{R}^{d}\right)\right)$ for every $T>0$.

Next, the authors consider the scalar conservation laws in bounded domains. Namely, one looks for $u \in L^{\infty}((0, T) \times \Omega)$ satisfying the following:

$$
\left\{\begin{array}{cc}
\frac{\partial u}{\partial t}+\nabla \cdot \mathbf{f}(u)=0, & \text { in }(0, T) \times \Omega \equiv Q_{T} \\
\left.u\right|_{\Gamma}=u^{D}, & \left.u\right|_{t=0}=u_{0}
\end{array}\right.
$$

where $\Omega \subseteq \mathbb{R}^{d}$ is a bounded smooth domain and $\Gamma=(0, T) \times \partial \Omega$. An immediate difficulty is that $u$ might be just an $L^{\infty}$ function whose trace on the boundary $\partial \Omega$ cannot be obviously specified. To overcome this, one introduces the following notion. A pair $(H, \mathbf{Q}), \mathbf{Q}=\left(Q_{1}, \ldots, Q_{d}\right), H, Q_{j} \in C^{2}\left(\mathbb{R}^{2}\right)$, is called a boundary entropy-entropy flux pair if for any $w \in \mathbb{R},(H(\cdot, w), \mathbf{Q}(\cdot, w))$ is an entropy-entropy flux pair and

$$
H(w, w)=0, \mathbf{Q}(w, w)=0,\left.\frac{\partial H(u, w)}{\partial u}\right|_{u=w}=0 .
$$

Then a function $u \in L^{\infty}\left(Q_{T}\right)$ is called a weak solution of (6) if (3) holds for all $\varphi \in C_{0}^{1}\left(Q_{T}\right)$, (5) holds for all entropy-entropy flux pairs $(\eta, \mathbf{q})$ in the distribution sense in $Q_{T}$,

$$
\underset{t \rightarrow 0+}{\operatorname{esslim}} \int_{\Omega}\left|u(t, x)-u^{0}(x)\right| d x=0,
$$

and for all $\beta \in L^{1}(\Gamma), \beta \geq 0$, almost everywhere, all boundary entropy-entropy flux pairs $(H, \mathbf{Q})$, it holds that

$$
\underset{s \rightarrow 0-}{\operatorname{esslim}} \int_{\Gamma} \mathbf{Q}\left(u(r+s \nu(r)), u^{D}(r)\right) \cdot \nu(r) \beta(r) d r \geq 0,
$$


where $\nu(r)$ is the outer normal of $\Omega$ at $r \in \partial \Omega$. Using the parabolic perturbation as in the Cauchy problem with much more careful analysis, the authors present the following result:

Theorem 2: If $\mathbf{f} \in C^{2}(\mathbb{R})^{d}, u^{D} \in L^{\infty}(\Gamma)$ and $u_{0} \in L^{\infty}(\Omega)$, then (6) has a unique weak solution.

In Chapters 3 and 4, the authors use the Young measure to discuss the sequence and its limits of approximate solutions to the scalar conservation laws obtained by the parabolic perturbation. This leads to a general framework of measure-valued solutions to nonlinear hyperbolic PDEs. Consequences are the existence of measurevalued solutions to the Cauchy problem for a scalar conservation law in one space dimension (with stronger condition on $\mathbf{f}$ and weaker condition on $u_{0}$ than Theorem 1 ) and to an initial-boundary value problem for a nonlinear wave equation (with the nonlinearity depending on the gradient of the solution).

In Chapter 5, the authors concentrate on the equations for a class of nonNewtonian fluids, or fluids with shear-dependent viscosity. For the incompressible fluid case, using the fundamental theorem on Young measures, the authors prove the global-in-time existence of measure-valued solutions. Further, the existence and uniqueness of weak solutions as well as strong solutions are also established under some stronger conditions. For the compressible fluid case, only the existence of measure-valued solutions has been proved. It is pointed out that the (global) existence of weak solutions to the compressible non-Newtonian fluids is still open.

This is a very well written book. It is very easy to read and pretty much selfcontained. One of the important contributions of the book is to make the recent work of F. Otto on the existence and uniqueness of weak solutions to scalar conservation laws in bounded domains accessible to a wide audience. This book probably will also be a very good introduction to the theory of conservation laws and measure-valued solutions to PDEs for graduate students. For those who are working in related areas, the book might also serve as a very nice reference.

\author{
WEAK AND MEASURE-VALUED SOLUTIONS TO EVOLUTIONARY PDEs \\ by J. Málek, J. Nečas, M. Rokyta and M. Ružička \\ Publisher Chapman \& Hall \\ USA: 115 5th Avenue, New York, NY 10003 \\ Publication Year 1996 \\ ISBN 0-412-57750-X \\ Price: $\$ 55.00$
}




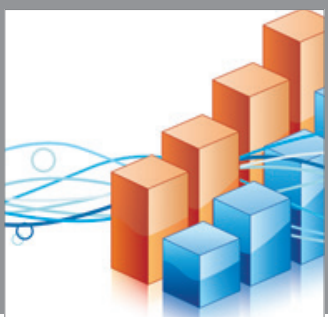

Advances in

Operations Research

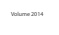

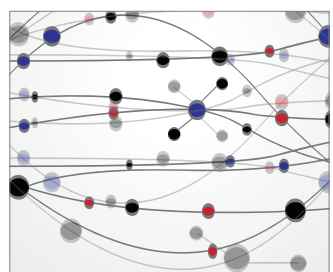

\section{The Scientific} World Journal
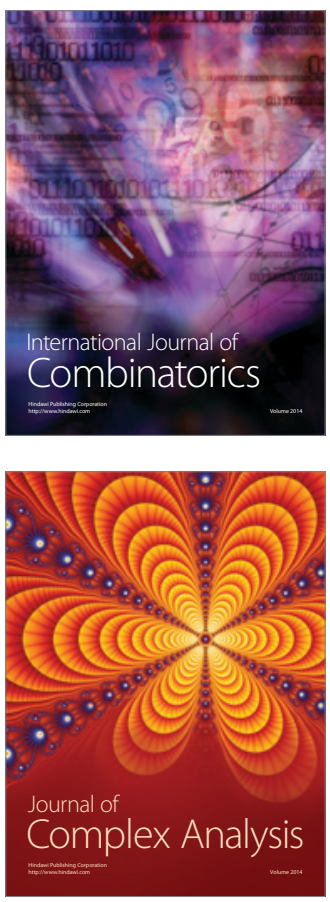

International Journal of

Mathematics and

Mathematical

Sciences
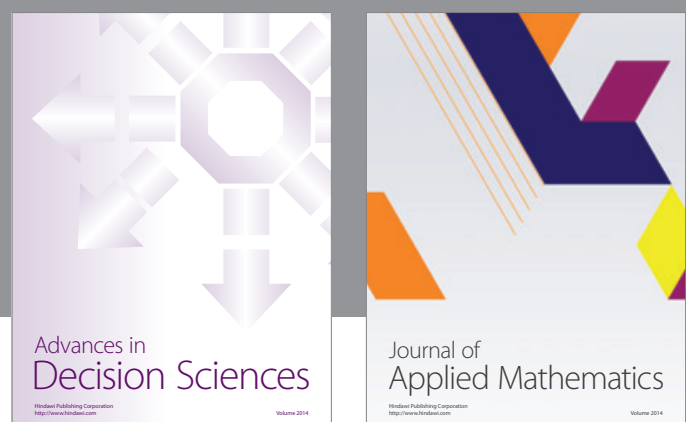

Journal of

Applied Mathematics
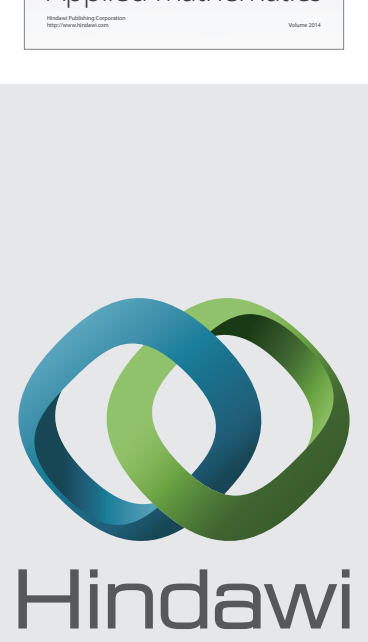

Submit your manuscripts at http://www.hindawi.com
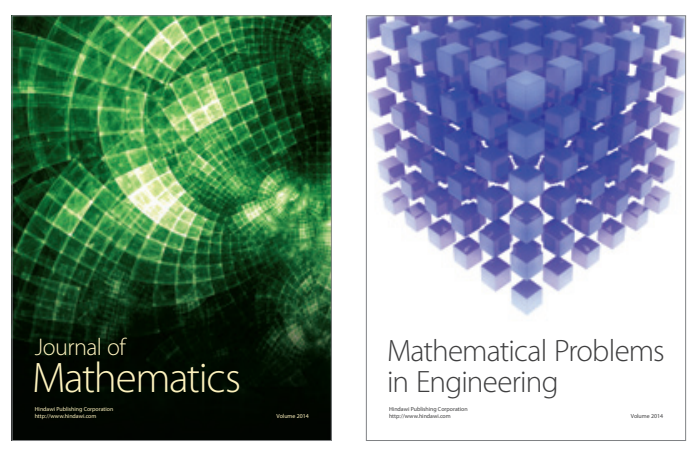

Mathematical Problems in Engineering
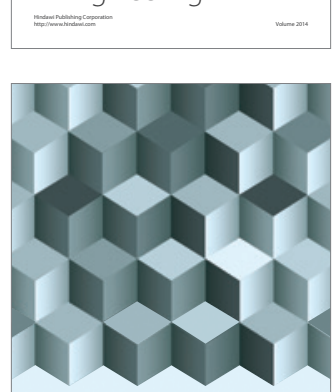

Journal of

Function Spaces
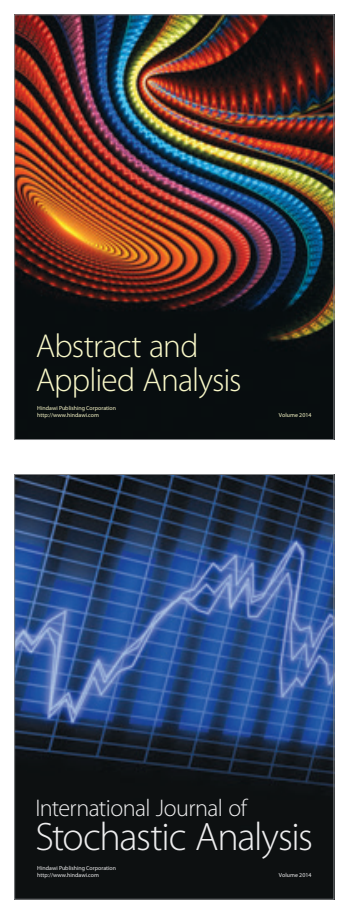

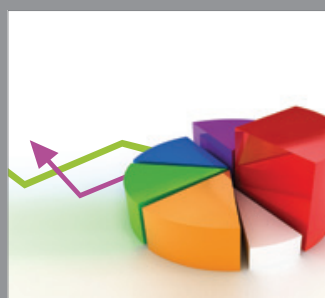

ournal of

Probability and Statistics

Promensencen
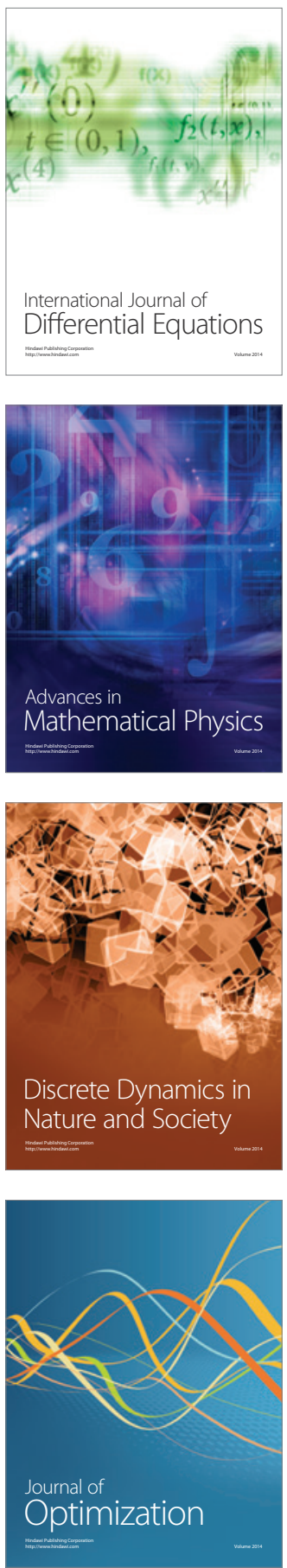